\title{
EVALUATION OF CHEST COMPUTED TOMOGRAPHY SCANS IN PATIENTS WITH SYSTEMIC SCLEROSIS USING THE WARRICK SCORE
}

\author{
lane Tamara Dondé1,^, Viviane Alves Costa ${ }^{1}$, Gizelle Gouvea Rezende ${ }^{1}$, Cristiano Michelini Lupo ${ }^{1}$, Vitor Amaro Muniz ${ }^{1}$ \\ 1.Faculdade de Medicina de São José do Rio Preto, São José do Rio Preto (SP), Brazil. \\ ${ }^{\star}$ Corresponding author: ianetamara@hotmail.com
}

\section{BACKGROUND}

Systemic sclerosis (SSc) is an autoimmune connective tissue disorder that is characterized by microvascular injury, excessive fibrosis of the skin and distinctive visceral changes that can involve the lungs, heart, kidneys, and gastrointestinal tract. Sclerodermarelated interstitial lung disease (SSc-ILD) is typically manifested by pulmonary function abnormality and changes on computed tomography scan (CT).

\section{METHODS}

Ten patients with systemic sclerosis who presented interstitial lung disease (ILD) and underwent the first pulse therapy with cyclophosphamide from 2015 to early 2020 were selected from our service. The patients included had a chest computed tomography (CT) prior to pulse therapy. Patients with overlapping or who had previously used mycophenolate mofetil and those who did not meet the ACR/EULAR 2013 classification criteria for SSc did not enter in the study. After completing the selection of patients, chest CT were individually assessed by a semiquantitative method using the Warrick score. This scoring system combines severity and extent of pulmonary involvement. The severity is graded from 0 to 15 according to different abnormalities. Extent is determined based on the total number of bronchopulmonary segments involved for each abnormality, ranging from 0 to 3 . These scores are combined to obtain a global score, ranging from 0 to 30 . All the patients were women, with an average age of 58.1 years and half were limited subset and half diffuse.

\section{RESULTS}

We observed that the average of the global score in the diffuse subset was 11.8 and 13.5 in the limited. The limited subset had a higher score in the severity, showing a variety of abnormalities with worse outcome, compared to the diffuse. As for the extension score, we did not observe significant variations (Table 1).

Table 1. Summary of the analysis of chest CT, according to Warrick score.

\begin{tabular}{cccc}
\hline Disease subset & Severity average & Extension average & Total average \\
\hline Diffuse (5) & 6.8 & 5.0 & 11.8 \\
\hline Limited (5) & 9.5 & 4.0 & 13.5 \\
\hline
\end{tabular}

\section{CONCLUSION}

Computed tomography scanning is an important technique for the detection and characterization of lung involvement in systemic sclerosis patients, in predicting treatment outcome, and assessing disease progression. Different systems for evaluating SSc-ILD on CT have been developed over the past 20 years. Semiquantitative scoring methods provide more precise assessment of quantity and type of ILD abnormalities. One scoring system that has been used in several studies was developed and published by Warrick et al. and it was used in our study.

\section{KEYWORDS}

Systemic sclerosis, Interstitial lung disease, Computed tomography scan.

\section{REFERENCES}

Assayag D, Kaduri S, Hudson M, Hirsch A, Baron M. High Resolution Computed Tomography Scoring Systems for Evaluating Interstitial Lung Disease in Systemic Sclerosis Patients. Rheumatology. 2012 Jan; S1:003. https://doi.org/10.4172/2161-1149.S1-003 